\title{
Online Teaching of Linear Algebra using webMathematica
}

\author{
http://dx.doi.org/10.3991/ijep.v2i3.2134 \\ E.M.Vorob’ev \\ National Research University Higher School of Economics, Moscow, Russia
}

\begin{abstract}
We discuss the online teaching of Linear algebra using the Wolfram Research software product called webMathematica. The teaching is based on interactive electronic tutorials developed by the author. The tutorials provide distant students with the instruments of remote calculation and visualization of the calculation results. All this increases the chances for students to deepen the understanding of the basic principles of Linear algebra and acquire the skills of solving problems.
\end{abstract}

Index Terms - Linear algebra, webMathematica, interactive electronic tutorial.

\section{INTRODUCTION}

The paper addresses the online teaching of linear algebra using web-based interactive electronic tutorials developed by the author. Mathematical disciplines, in general, and linear algebra, in particular, require the development of problem solving skills. Without solving problems, the assimilation of the discipline by students cannot be regarded as deep and effective. While it is simple to present the background of the mathematical discipline on the Internet, just by putting an electronic version of the textbook on the website, problem solving should be interactive. The web site should be supplied with instruments which students can use to perform remote calculations. At the same time, the teacher should be able to assess them on-line by using webinars, chats, forums, and blogs. We use webMathematica[1] as a software system that provides computing and teaching on the Internet.

WebMathematica is a web technology for doing symbolic, graphic and numerical remote calculations based on scientific and educational interactive electronic documents hosted on a Java server. The distant students use only web browsers such as Internet Explorer or Netscape for calculations.

WebMathematica can be regarded as a web interface for the computational engine (the kernel) of the computer algebra system (CAS) called Mathematica. Note that there may be several Mathematica kernels on the server. But Mathematica is deeply hidden from users who only need to know the Mathematica input syntax for the mathematic formulae the users enter.

\section{IMPACT OF COMPUTER ALGEBRA SYSTEMS ON MATHEMATICS INSTRUCTION}

Computer algebra systems have been used in education since their first appearance in the 1980s. Educators used them both for lectures and for computer labs to enhance the teaching of Calculus and related courses. Mathematica is attractive for teaching because its users work with elec- tronic documents called Notebooks in which text and executable commands coexist. This is very important since educators can easily write interactive tutorials for students. These tutorials make mathematics more exciting for students by getting them personally involved in experimentation and discovery.

The influence of computer algebra systems on the content and teaching methods of mathematical disciplines is carried out in several directions. Computer algebra systems provide more detailed and profound discussion of the basic concepts and principles of the mathematical disciplines than is possible within traditional instructional techniques, because of the time they save that instructors usually devote to performing routine and cumbersome calculations and drawing function graphs when delivering lectures in classes. CAS can do this job quickly and accurately. At the same time, instructors can dwell on numerous examples of direct interest to students and problems which are relevant for the "real world". As a consequence, the exposition of disciplines becomes more accessible and compelling, and students have fun learning.

If, in addition, students can attend computer classes at any time or they have CAS installed on their personal computers or laptops, they have a good opportunity to study the theory and to solve problems with the aid of arbitrarily patient mentors in the form of interactive tutorials.

We refer the reader to [2] for further details.

\section{WHY WEBMATHEMATICA}

WebMathematica was chosen for the following reasons. The author had intensively used CAS Mathematica for teaching students in computer classes for a long time. To do this, the electronic tutorials containing Linear algebra background, exercises, and programs written in a program language "Mathematica" were developed. Once Wolfram Research released webMathematica, it was natural and not difficult to translate Mathematica tutorials into webMathematica ones.

It was timely, since my colleagues not familiar with Mathematica met with certain difficulties when teaching Linear algebra in computer classes. The same could be said for students. WebMathematica completely eliminated these kinds of obstacles. However, to more successfully usewebMathematica, it is desirable to develop program tools that would allow the users to enter the mathematical formulae in the usual mathematical form, i.e. with special symbols for quotients, powers, roots, sums, derivatives, integrals, series, etc.

Another reason for adopting webMathematica is the spread of e-learning, which requires providing access to 
interactive educational resources for distant students. Currently, distance students of the Higher School of Economics use the educational portal http://miem.iedu.ru to gain access to webMathematica as well as to receive problems to solve, teacher comments and assessments.

The webMathematica operation also showed that fulltime students readily use it to prepare for tests and exams. In fact, the access of students to their teachers is limited due to the lack of teacher time, while webMathematica allows unlimited time to practice and understand basic concepts of Linear algebra.

Some other issues of the webMathematica application for learning mathematics are discussed in [3].

\section{BRIEF REVIEW OF WEBMATHEMATICA}

We recommend the reader who knows nothing about webMathematica to visit the web site http://library.wolfram.com/explorations/ to become familiar with it. There, one can interact with a number of examples of webMathematica applications.

WebMathematicacreates special web pages that contain HTML-forms with input fields for entering user information. These forms can be supplied with radio buttons and popup menus. The pages are then sent to the user upon request.

WebMathematica, installed on the Java server, uses two standard Java technologies: Java servlets and Java server pages. But the developer of webMathematica tutorials does not need to know anything about Java except for several special tags that work with Mathematica. Mathematica's commands and programs are placed between <msp:evaluate $>$ and </msp:evaluate $>$ tags. WebMathematica sends these commands for execution to the Mathematica kernel. The results of calculations are inserted into the generated new web page.

Now, we must mention some things which have important implications for the authors of interactive tutorials. The <msp:evaluate $>$ tag extracts one of the Mathematica kernels from the kernel pool. This kernel then makes a calculation and the </msp:evaluate $>$ tag clears the kernel and sends it back to the pool. As a result, no information about the calculations made remains in the kernel. This makes it impossible to use the usual Mathematica mechanism of assigning global values to the variables. Instead, it is necessary to declare such a variable as a session variable. The value of the session variable is stored on the server or on the user's computer.

After the user fills in the input fields with mathematical formulae, he/she presses the Calculate button, the page goes back to the server, the formulae are calculated, and the results of calculation are pasted onto a new web page resent to the user.

It should be noted that it is impossible to get direct access to the computing engine of Mathematicawith the help of webMathematica. The user can execute only those commands that are contained in the body of the HTMLforms. Thus, only a relatively small set of the Mathematica commands are available for the user on each web page. This significantly limits the flexibility of webMathematica and forces the instructor to invent new teaching methods to overcome these limitations.

\section{BENEFITSOF TEACHING WITH WEBMATHEMATICA}

We would like to mention that webMathematica offers various benefits to users who are teaching mathematical science. In first place, we have the high-quality static and dynamic two- and three-dimensional graphics. These graphics can be animated and rotated. Graphical objects constitute the core of visualization inmathematics.

Furthermore, we can mention the unmistakable automation of cumbersome symbolic and numeric calculations. Additionally, webMathematica offers important interactivity (i.e. solving the problems for which data are not known by the authors of electronic tutorials). Finally, it can be noted the verification of calculations performed with pencil and paper with webMathematica is very useful for students. The following principle for using webMathematica is adopted in the interactive electronic tutorials developed by the author.

It is clear that it would be pointless to write programs that completely automate calculations made by students in order to obtain the answer to problems they solve, although this is possible. Therefore, interactive tutorials should provide detailed examples of solving problems with calculations that are performed both manually and through webMathematica. Students repeat these calculations with respect to their problems either on the server or by using traditional pen and paper.

\section{INTERACTIVE ELECTRONIC LINEAR ALGEBRA TUTORIALS}

Our online teaching of Linear algebra by using webMathematica is based on nine interactive electronic tutorials developed by the author. The tutorials are available on the web site http://wm.iedu.ru. Here are their titles: Echelon forms of matrices, Determinants, Inverse matrix, Systems of linear equations, Linear spaces, Linear maps, Jordan's basis of the linear map, Euclidean spaces, and Quadratic forms.

The content of the Echelon forms of matrices, Determinants, Inverse matrix, and Jordan's basis of the linear map tutorials is clear from their titles. The Systems of linear equations tutorial considers the application of the GaussJordan method for solving the systems of linear algebraic equations.

The Linear spaces tutorial is devoted to checking the linear independence of the vector systems and to calculating the transition matrices. The tutorial called Linear maps deals with two types of problems. The first is the calculation of the linear map matrices and their transformation under the transition to a new basis. The second type is the calculation of the eigenvalues and the eigenvectors of the linear map. The map is given by its matrix.

There are also two topics in the Euclidean spaces tutorial. The first is the Gram-Schmidt algorithm for obtaining the orthogonal basis and the second is calculation of the systems of the orthogonal Laguerre, Chebyshev, Lagrange, and Hermite polynomials.

We consider the application of the Lagrange algorithm in the Quadratic forms tutorial.

Figure 1 shows the main page of the first in our list of the tutorials. This tutorial considers the topic "Echelon forms of matrices”. 


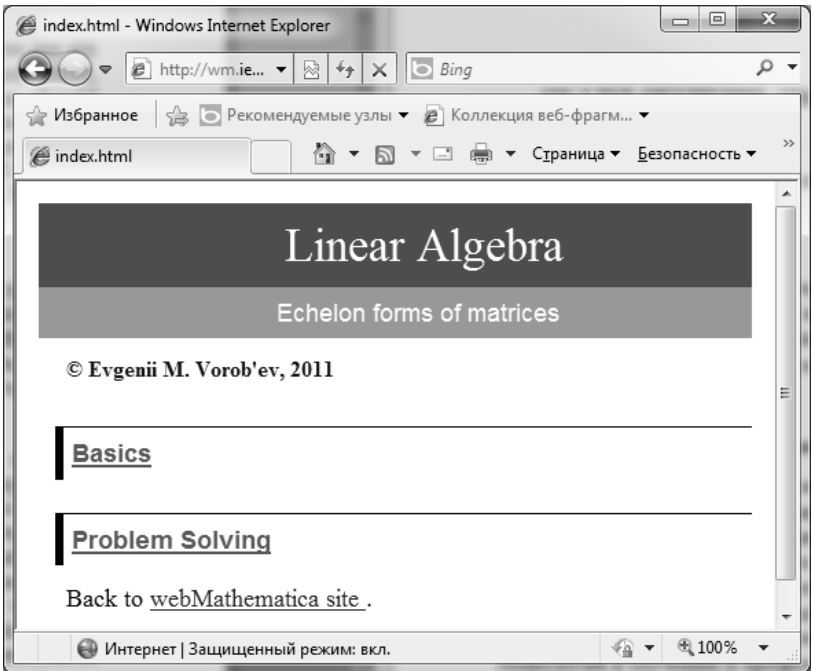

Figure 1. The main page of Linear Algebra tutorial

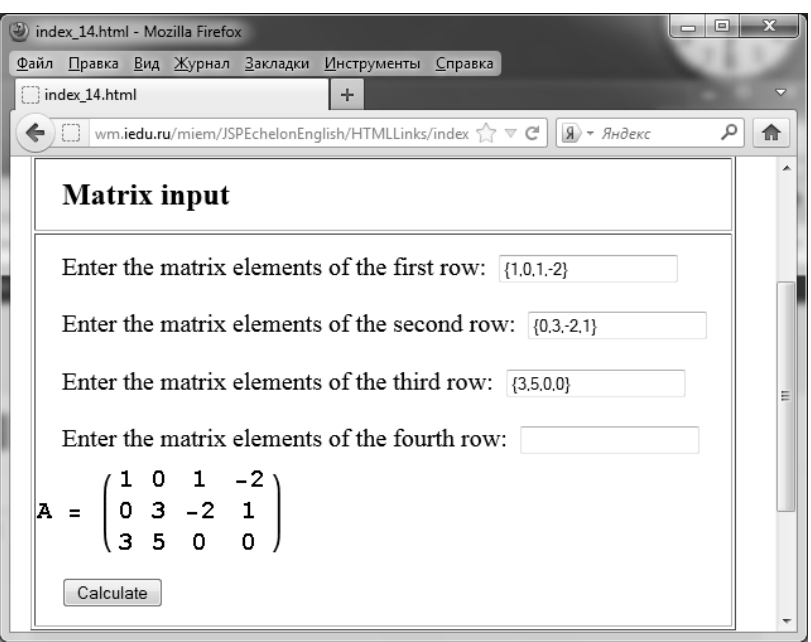

Figure 2. HTML-formcalled Matrix input

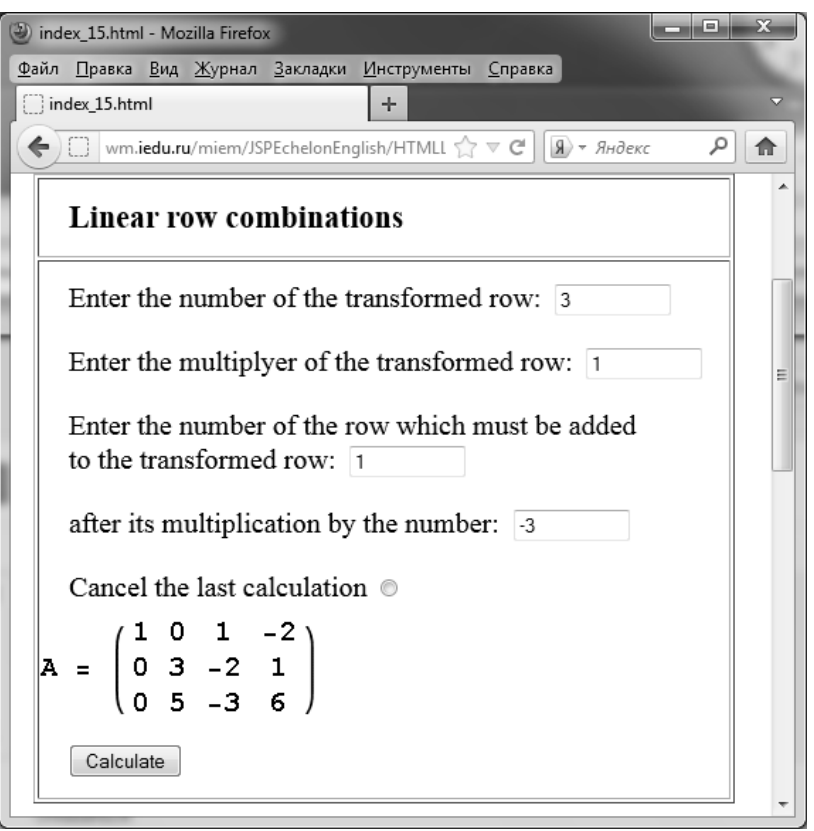

Figure 3. Linear row combinations HTML-form
The visual appearance of the main page is typical for all the tutorials. Each of the main pages contains hyperlinks to the basics and to problem solving with the help of Mathematica.

If we open the section "Problem Solving, we can see four subsections: "Problems," "Reducing to the upper echelon form. Pattern of problem solving", "Reducing to the lower echelon form. Pattern of problem solving", and "Calculation instruments". We begin by considering the last.

\section{A. Calculation instruments}

As is well known, reducing matrices to the echelon forms uses two types of row transformations; more exactly, linear combinations of rows and row permutations. In addition, the implementation of reducing the matrix to the echelon forms by using webMathematica involves the input of the matrix to the computational kernel of Mathematica. This constitutes the initial step of the calculation. It is executed using the HTML-form called Matrix input (see Figure 2).

Matrices should be input row by row. The Matrix input HTML-form provides how to enter the matrices, which may consist of up to four rows. The input of matrices with three columns and two rows is also possible. To do this, users can leave one or two of the last input fields blank. Figure 2 shows the state of the HTML-form after pressing the Calculate button. The matrix entered is denoted by $\mathrm{A}$ which is declared as a session variable.

The Matrix input HTML-form is placed on a separate web page since the operation mechanism of webMathematica involves the calculation of all the HTML-forms on the web page, no matter what form is submitted for computing by the user. The user can press only one Calculate button on the web page. Suppose that there is another HTML-form on the web page, the form which calculates, for example, the permutation of matrix A rows. If the user should press the Calculate button of this latter form, the value of A for this form would be Null.

The HTML-form, called Linear row combination, calculates the linear combinations of the matrix A rows (see Figure 3). It is placed on the web page entitled Common Step.

In order to calculate the linear combination, we enter the number of the transformed row, the number of the auxiliary row, and their multipliers. After having done the input, we press the Calculate button and receive the result shown in Figure 3. The latter shows the result of calculation of the linear combination of rows 3 and 1 with multipliers 1 and -3 . This linear combination is substituted for the third row of the matrix. If the student makes a mistake, he/she has the opportunity to cancel the last calculation by pressing the radio button.

Sometimes it is necessary to permute the matrix rows. For this purpose, we created the Permute rows HTMLform located at the same web page as the form Linear row combination.

\section{B. Problems}

The subsection "Problems" contains ten problems involving the matrices with four rows. Here is a typical matrix from the subsection (see Figure 4): 


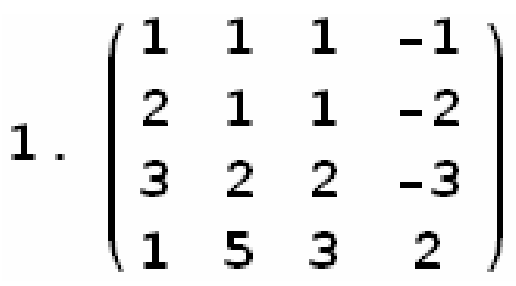

Figure 4. Matrix to reduce to the echelon forms

The student should reduce the matrix to the upper and lower echelon forms. Then, after reducing, he/she should calculate its rank as the number of nonzero rows.

\section{Patterns of problem solving}

On-line learning does not involve face-to-face contact with the teacher. Therefore, students should become familiar with webMathematica computing exclusively by using the tutorials. That is why we provided the tutorials with step-by-step instructions for solving problems. These instructions are illustrated with the HTML-forms containing the data filled input fields. The data are taken from the problem considered as an example and entered by the author of the tutorial. The data cannot be changed by the user. The forms which are located in the Patterns subsection visually look like the forms shown in Figures 2 and 3.

There might be an alternative way to create the problem solving patterns. The author could permit the students to edit the input fields of the HTML-forms as is done in the Calculation tools subsection. But there are subtleties. Each problem requires its own number of steps to be solved in contrast to most Calculus problems. In the pattern, each step must be performed using its own HTML-form. Each of the forms must be placed on its own web page, but this is impossible.

\section{E-PEDAGOGY OF THE TUTORIALS}

It is obvious that e-learning technologies imply the use of new pedagogies [4], since the latter considers the process of teaching and learning in a more social environment. The learning environment has changed significantly over the past twenty years. Computers and the Internet have provided new tools for teaching and learning, and societal changes require persons to seek continuous education throughout their lives. We have chosen not consider such general issues in this paper. We focus, instead, on a very specific pedagogical problem of how to teach using webMathematica.

We share the main pedagogical principle of webMathematica-based learning. The principle best expressed as: "to learn mathematics not calculation", or "math calculating”. Interactive dynamic facilities, which are HTML-forms, play a crucial role in our tutorials. They allow students to concentrate on the basic ideas of Linear algebra by making the majority of the calculations. In the previous section, we dwelt on this issue when considering the echelon forms of the matrices. The same release from user-made calculation takes place in each of the tutorials.

The Echelon forms of the matrices tutorial considered in the previous section clearly demonstrate our pedagogical principle: to provide students with the opportunity to focus on the essence of the mathematical method by automating the arithmetic calculations. This automation takes place in each of the nine tutorials. For example, when calculating the eigenvalues of the linear maps as

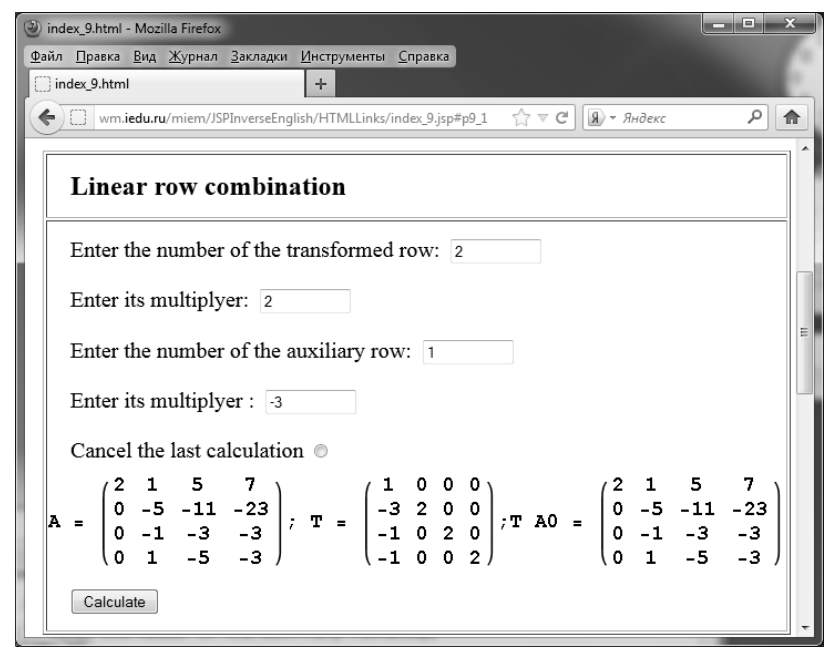

Figure 5. Results of the intermediate calculation

roots of the characteristic polynomial, the students receive the factored characteristic polynomial from webMathematica. The same is true with respect to calculating the eigenvectors since WebMathematica provides the linear algebraic equations for the components of the eigenvectors.

When making the polynomials orthogonal, we automate the calculation of the definite integrals which are the scalar products of the polynomials. When applying the Lagrange method to reduce the quadratic form to the canonical form, we automate the completing the square with respect to one of the variables and the computation of the quadratic form after the change of the variables.

Obviously, the visualization prevailing in Calculus does not play a key role in Linear algebra. Nevertheless, its importance cannot be underestimated. We resort to the visualization of final and intermediate results in all of our tutorials. The visualization of intermediate results helps students understand the Gauss-Jordan method of calculating the inverse matrix. Indeed, the product $\mathrm{T}$ of the matrices of the consecutive elementary matrix row transformations is a key object of the method. After the matrix is reduced to its diagonal form by elementary transformations, the matrix $\mathrm{T}$ is equal to the inverse matrix. Figure 6 shows the HTML-form called "Linear row combination" with the results of an intermediate calculation. The transformed matrix $\mathrm{A}$, the matrix $\mathrm{T}$ and the independently calculated product of $\mathrm{T}$ and the initial matrix $\mathrm{A}$, denoted as A0, are depicted in this Figure.

\section{VIII.CONCLUSIONS}

The distance students of Moscow Higher School of Economics actively used the interactive electronic tutorials presented here in the fall and spring semesters of the 2011/2012 academic year. Although the tutorials contain the necessary minimum of theoretical background, the Linear algebra textbook was also available on the website.

Online learning demonstrated the positive effect of the tutorials. The students successfully passed the exams and confidently solved problems. They did not encounter any problems working with the tutorials. This shows that webMathematica can effectively help teach young people who have long adapted to the Internet. 
PAPER

ONLINE TEACHING OF LiNEAR ALGEBRA USING WEBMATHEMATICA

\section{REFERENCES}

[1] Tom Wickham-Jones. WebMathematica: A user Guide. Wolfram Media, 2001.

[2] T. Guyer. "Computer Algebra Systems as the Mathematics Teaching Tool.” World Applied Sciences Journal, vol. 3 (1), 2008, pp.132-139.

[3] G. Albano, B. D’Auria, and S. Salerno. “A webMathematica Application for Mathematics Learning” in Lecture Notes in Computer Science, 2657, P.M.A. Sloot et al. Eds. Berlin-Heidelberg, Springer-Verlag, 2003, pp. 754-763.
[4] M. Smith. "An Investigation into the Process of E-Pedagogy and E-Learning in an Online Community.” Reflecting Education, v.1, No. 1-2, 2005, pp. 9-23.

\section{AUTHOR}

E.M. Vorob'ev is with the National Research University Higher School of Economics, 3/12 B. Trekhsviatiyelskii, Moscow 109028 Russia (e-mail: emv@miem.edu.ru).

Received 29 Mai 2012. Published as resubmitted by the author 24 June 2012. 